(17) K. Burns, J. Opt. Soc. Amer. II, $301,1925$.

(I8) Trans. I.A.U. 5, 30I, I935.

(19) R. Ritschl and H. Schober, Physik. Z. 38, 6, 1937.

(20) W. F. Meggers and C. J. Humphreys, J. Res. B.S. 13, 293, I934.

(2I) T. A. Littlefield and D. T. Turnbull, Proc. Roy. Soc. A, 218, 577, 1953.

(22) K. Burns and K. B. Adams, J. Opt. Soc. Amer. 43, 1020, I953.

(23) C. J. Humphreys, J. Res. N.B.S. 20, 17, 1938.

(24) T. A. Littlefield, Proc. Roy. Soc. A, 187, 220, x946.

(25) E. Rasmussen, Rydberg Cent. Conf., Lund, 1954.

(26) E. Engelhard, P.V. Com. int. Poids Mes. 2e ser. 23, I65.

(27) H. Barrell, Proc. Roy. Soc. A, 209, 132, 1951.

(28) K. Burns and K. B. Adams, J. Opt. Soc. Amer. 42, 56, 1952.

(29) G. V. Deverall, K. W. Meissner and G. J. Zissis, Phys. Rev. 95, I463, 1954.

(30) G. Righini and M. Rigutti, Osservaz. mem. Arcetri, 69, I23, 1954.

(3I) M. G. Adam, Monthly Notices, Ir2, I49, 1952.

(32) S. Glad, Ark. Fysik. 7, 7, I953.

(33) K. Bockasten, Ark. Fysik. 9, 457, 1955.

(34) R. A. Fisher and F. E. Eshbach, J. Opt. Soc. Amer. 43, ro3o, 1953.

(35) P. Risberg, Ark. Fysik. 9, 483, 1955.

(36) B. Edlén and P. Risberg, Ark. Fysik. ro, 553, 1956.

(37) C. C. Kiess, J. Res. N.B.S. 5I, 247, 1953.

(38) C. C. Kiess, J. Res. N.B.S. 47, 385, $195 \mathrm{I}$.

(39) S. Glad, Avk. Fysik. ro, 291, 1956.

(40) A. G. Shenstone, J. Opt. Soc. Amer. 44, 749, 1954.

(4I) C. C. Kiess, J. Opt. Soc. Amer. 43, 1024, I953.

\title{
14a. SUB-COMMISSION ON INTENSITY TABLES: REPORT ON TABLES OF $f$ VALUES
}

The necessity of collecting all available oscillator strengths, either determined by experiments or calculated by quantum mechanics, has been repeatedly emphasized in these Transactions. A first survey of the literature was published in the report of Commission 36 for the I $94^{8}$ meeting. Since then, several such tables have been prepared, which apparently will soon provide for this generally felt need.

(a) In I950 appeared the sixth edition of Landolt-Börnstein's Zahlenwerte und Funktionen, of which Vol. I contains tables of $f$ values by Biermann. The tables of absolute values are fairly complete; relative values are quoted for $\mathrm{Fe}$ and $\mathrm{Ti}$; for other elements the necessary references are given. Special lists refer to forbidden lines and to molecular bands. Dr Biermann writes that he is still keeping his catalogue up to date in view of further editions.

(b) In the book by Allen, Astrophysical Quantities (in the Press) about 350 oscillator strengths of astrophysical importance will be collected, Ioo of these being components of hydrogen lines.

(c) Another list is being prepared by Menzel for the Smithsonian Physical Tables.

(d) In the new edition of Unsöld's book, now in the Press, a very complete list of references will be communicated, ordered according to the periodic system and mentioning whether the determinations were absolute or relative, experimental or theoretical.

(e) Finally, a list is expected to be found in Condon, Handbook of Physics, part 7 , chap. 3 (in the Press; McGraw Hill).

Although these compilations will be of the greatest use for the astrophysicist, it must be recognized that they do not yet give the whole of the data, critically combined and ready for use. No spectroscopist would be satisfied if instead of tables of spectral lines he had only a list of references or the wave-lengths of 350 selected lines. There is therefore ample opportunity for future work, which should make available all theoretical and 
experimental data from various sources, critically balanced and combined. For the astrophysical investigation, there is also more and more need of other data on the individual spectral lines, which partly determine their strength and their width in cosmic objects: (I) the line broadening and the asymmetry due to the Stark effect; (2) the hyperfine structure. It would be extremely useful if it was possible to collect such quantities, now scattered through the literature.

In this report a review will be given only of the most recent work on $f$ values, done since the I95I I.A.U. meeting, and not always easily accessible, because the publications are distributed between physical and astronomical periodicals. These references have been collected by an active collaboration of the President of Commission I4, of the members of the sub-commission and of other scientists. A small list has been added of Russian determinations of recent years, which seem to be hardly known in the Western countries, and have been quoted mainly from the paper of Mitrofanova.

\section{Theoretical investigations}

The very useful paper of Bates and Damgaard, allowing a quick calculation of atomic transitions in first approximation, has been followed by a similar paper of Bates on molecular transitions for all diatomic molecules. By taking as a starting-point the observed spectroscopic constants, the calculation could be made very simple.-Of interest is a sum rule for molecular transitions, due to Ter Haar.-Pasternack, Shortley, Aller, Baker, Menzel and Gottschalk had already shown that calculations assuming the Russell-Saunders coupling can be considerably improved if intermediate coupling is taken into account, this meaning the interaction between the electron spin with its own orbit. Garstang and others applied the method to other cases and reached a similar success. Still better results can be obtained by considering the spin-spin interaction between the two electrons and the spin-other orbit interaction; this has been done earlier by Aller, Ufford, van Vleck and Gilmour, and has been applied recently by Garstang (I95I) to several atoms and ions. The calculation of the effect of configuration interaction would give the best results, but at the cost of increased labour. Layzer has recently developed a new method for the problem of configuration mixing and has applied it to some special cases, e.g. to $\mathrm{Caxv}$.

Menzel and Layzer remark that the energy levels are insensitive to small errors in the wave functions, whereas the $f$ values are not. This considerably increases the difficulty in predicting $f$ values.

\section{Experimental determinations}

An extensive investigation was undertaken by Meggers and his collaborators. They measured under standard conditions the intensities of 30,000 spectral lines, belonging to 70 elements, between 2000 and $9000 \AA$, and proved that their results did correspond to the multiplet rules and other known $f$ values. When published, these determinations will be of great value to the astrophysicist, though they are admittedly rough.

As to the absolute measurements, several methods have been applied which do not require a knowledge of the vapour pressure of elements at high temperatures-the weak point in much of the earlier work.

I. The method of anomalous dispersion of Rozhdestvenski was taken up with remarkable success by his pupils and applied even to elements of small volatility.

2. Stephenson measured the magnetic rotation.

3. Kopfermann and Wessel applied the method of the atomic beam to the important case of iron. The absolute values thus found are about three times greater than those previously assumed; a factor of that order had already been suspected earlier. The same method is now being applied by $\mathrm{R}$. $\mathrm{B}$. King to $\mathrm{Fe}, \mathrm{Cr}$ and $\mathrm{Cu}$.

4. Huldt and Lagerquist spray in a flame known quantities of a salt.

5. In the Physical Laboratories at Kiel and at Erlangen, an electric arc is stabilized 
by the whirling motion of the surrounding gas; several liquids may be injected tangentially. The arc is observed end-on, and its temperature may reach $50,000^{\circ}$.

6. At the Michigan Observatory, the luminous shock tube is used for the investigation of $f$ values, in particular for $\mathrm{Ne}$.

For the astrophysically important elements $\mathrm{Fe}, \mathrm{Mn}, \mathrm{Ni}$ and $\mathrm{Cr}$, absolute probabilities have now been determined. It is to be hoped that soon measurements will be obtained for $\mathrm{Co}, \mathrm{Ti}$ and $\mathrm{V}$, and for the ionized atoms of these seven elements.

\section{Astrophysical determinations}

A derivation of $f$ values from curves of growth has been tried several times. The results are valuable, since they often refer to lines which could be investigated only with difficulty in a laboratory, but they may show considerable mean errors, for the line formation in a stellar atmosphere is a very complicated phenomenon.

A paper by Aller and his collaborators gives values for Fe II, TiII, $\mathrm{Cr}$ II and some other ionized elements, derived from the spectrum of XX Oph. (I954, in the Press).

\section{Nomenclature}

Data on transition probability are expressed in various ways: $A, f, g f, S$. Menzel and Layzer suggest using as a rule the line-strength $S$ (definition, cf. Condon and Shortley, p. 98). This is closely related to the other quantities mentioned above; it has the advantages: (I) to be the most useful from a theoretical point of view; (2) to satisfy certain sum rules; (3) to be symmetric in the initial and final levels.

Others object that transitions with comparable line-strengths have very different intensities, if electric dipole, electric quadrupole and magnetic dipole radiation are compared. They prefer the products $g f$, which are also symmetric.

A short discussion at the next I.A.U. meeting would be useful.

\section{Minnaert \\ President of the Sub-commission}

\section{General experimental papers}

\section{BIBLIOGRAPHY}

Stephenson: Proc. Phys. Soc. Lond. A, 64, 458, 1951.

Ditchburn and Heddle: Proc. Roy. Soc. A, 220, 61, 1953 (contin. abs.).

\section{General theoretical papers}

Green, Weber, Krawitz: Ap. J. Ir3, 690, I95I.

Horie: J. Phys. Soc. Japan, 7, 58, 1952.

Kessler: C.R. Acad. Sci. Paris, 236, I 335, 1953.

General theoretical papers on forbidden lines

Naqvi: Astr. $J \cdot 56,45$, I95I.

Osterbrock: $A p . J$. Ir4, 469, I9.5I.

Yamanouchi and Horie: J. Phys. Soc. Japan, 7, 52, I952.

\section{Atomic transition probabilities}

$\begin{array}{ll}\text { A II } & \text { Garstang: } M . N . \text { Ir4, II8, I954. } \\ \text { Ag I } & \text { Terpstra: Diss. Utrecht (in the Press). } \\ \text { Ba I } & \text { Wessel: } Z \text {. Phys. r3o, I06, I95I (correction). } \\ \text { CI, CII } & \text { Maecker: } Z \text {. Phys. I35, I3, I953. } \\ \text { CaI } & \text { Trefftz: } Z \text {. Ap. 29, 287, I95 I. } \\ & \text { Green, Weber, Krawitz: Ap. J. Ir3, 690, I95I. } \\ & \text { Olsen, Routly, King: Indiana Univ. Conference, I954, p. } 46 . \\ & \text { Biermann: Rydberg Conference, I954. }\end{array}$




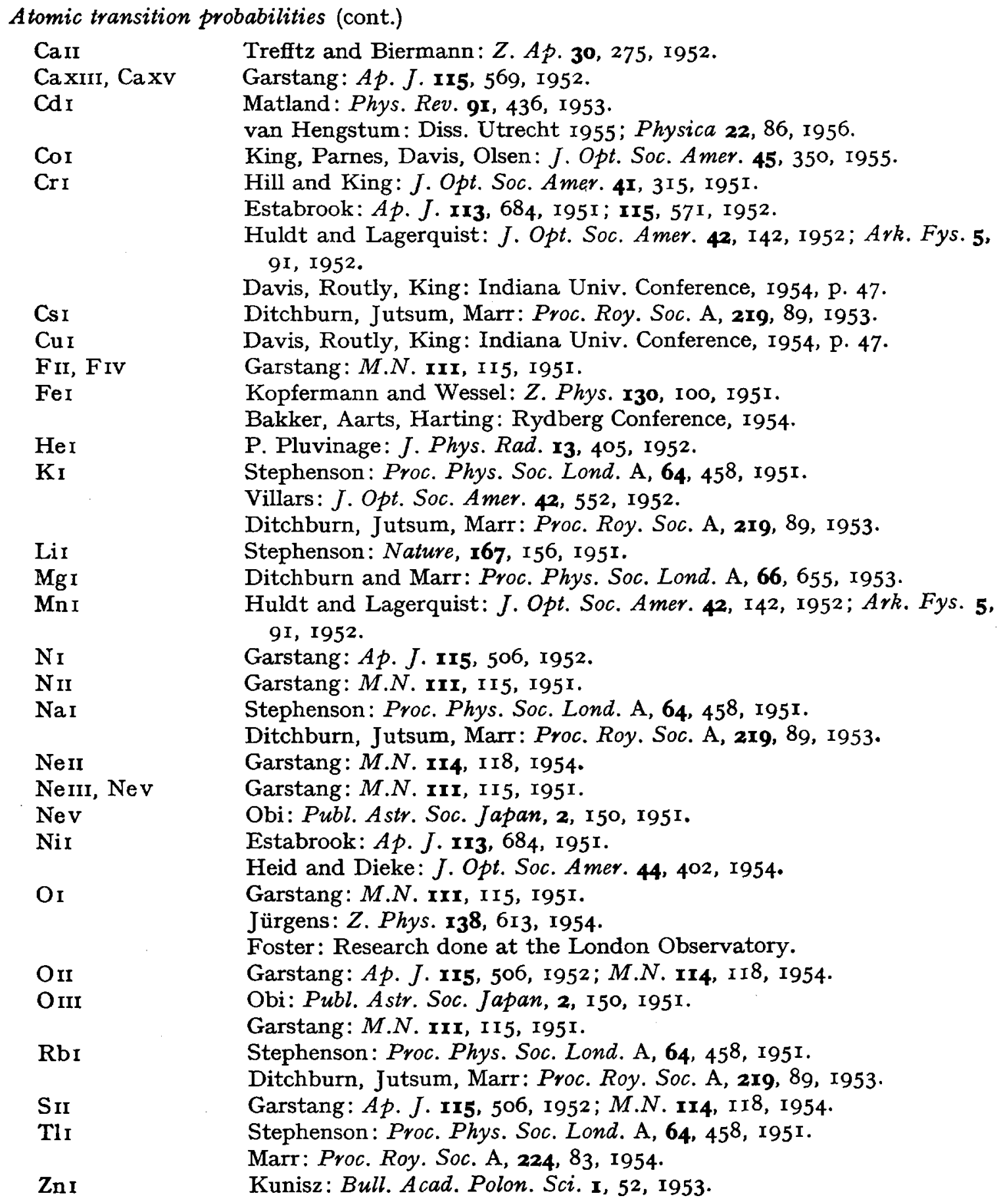

\section{Russian papers}

General

General

BaII

BiI

CrI

CrII

Veselov: J. Exp. Theor. Phys. I19, 959, 1949.

Vaïnshteïn and Yavorski: C.R. Acad. Sci. U.R.S.S. 87, 919, 1954.

Penkin: J. Exp. Theor. Phys. 17, III4, 1947.

Sobolev: J. Exp. Theor. Phys. 13, 1943, or 19, 1949.

Penkin: Bull. Acad. Sci. U.R.S.S., Ser. phys., 11, $217,1947$.

Zagorianskaya: Bull. Acad. Sci. U.R.S.S., Ser. phys., 19, 447, 1949 J. Exp. Theor. Phys. 19, Nr. 5, 1949. 

Fer, FeII
Sobolev: J. Exp. Theor. Phys. 13, I943, or 19, 1949.
Mitrofanova: Izv. Pulkovo Obs. 19, Nr. 2, I952.
$\mathrm{NaI}$
Kvater: Vestnik Univ. Leningrad, Nr. 2, 135, 1947.
$\mathrm{Tl}$ I
Kvater: Vestnik Univ. Leningrad, Nr. 2, 135, 1947.

Some Molecular transit
General
General
General
$\mathrm{H}_{2}{ }^{+}$
$\mathrm{OH}$
$\mathrm{O}_{2}$

$\mathrm{~N}_{2}+$
$\mathrm{C}_{2}$
$\mathrm{CN}^{2} ; \mathrm{N}_{2}+$
$\mathrm{C}_{2} ; \mathrm{N}_{2}+\mathrm{CN} ; \mathrm{BO}$
$\mathrm{CH}_{4} ; \mathrm{CO}_{2}$

Molecular transitions of astrophysical importance

Pillow: Proc. Phys. Soc. Lond. A, 64, 772, 195 I.

Bates: M.N. 112, 614, 1952.

Ter Haar: Proc. Roy. Soc. Edinb. A, 63, pt IV, 381 , I952.

Bates et al.: J. Chem. Phys. 19, 1122,1951 ; Proc. Phys. Soc. Lond. A, 66, 784 and 1124,$1953 ; A, 67,533,1954$.

Penner: Cal. Inst. Techn., Contract Nonr-220(o3)-NR or540I.

Dyne: Cal. Inst. Techn., Contract Nonr-220(03)-NR or 5210.

Pillow: Proc. Phys. Soc. Lond. A, 66, 733 and 1064, 1953; 67, 847, 1954.

Fraser and Jarmain: Proc. Phys. Soc. Lond. A, 66, I I 45 and I I 53, 1953.

Pillow: Proc. Phys. Soc. Lond. A, 67, 780, 1954.

Jarmain and Nichols: Canad. J. Phys. 32, $201,1954$.

Jarmain, Fraser, Nichols: Univ. Western Ontario, Dept. Phys., Contract Nr. A.F. 19 (122), 470, 1953 and 1954.

Floyd and King: Proc. Phys. Soc. Lond. A, 64, 209, 1951.

Turner and Nicholls: Phys. Rev. 82, 290, 195I.

McKellar and Tawde: $A p . J . \times 13,440,195$ I.

Floyd and King: Indiana Univ. Conf. 1954, p. 44.

Shull: $A p$. J. 1r4, 546, 1951 .

Pillow: Proc. Phys. Soc. Lond. 64, 772, 1951.

Goldberg: in Kuiper, The Earth as a Planet, ch. 9, with review of recent literature.

\section{Report of meeting. 3I August I955}

President: M. B. Edlén.

SeCretary: Mme C. Moore-Sitterly.

The President discussed item by item the printed Draft Report of the Commission. With regard to the Primary Standard, he pointed out that in adhering to the present recommendation, Commission $\mathrm{I}_{4}$ will cease to be the ultimate authority if and when the primary standard of wave-length becomes identical with the primary standard of length. The question is now referred to the International Committee on Weights and Measures. Commission I4 passed a resolution recommending that the I.A.U. endorse the proposals regarding the future primary standard of wave-length, made by the Advisory Committee for Re-defining the Metre and stated in the Draft Report, p. 2or of this volume.

It was agreed that the Dispersion Formula proposed by Edlén in 1952 represents the best present knowledge of the index of refraction of air, although it is extrapolated for wave-lengths longer than $6438 \AA$. Essen pointed out that this formula fits present measurements in the microwave region within the errors of observation. The Commission passed a resolution that the I.A.U. recommend for the conversion of wave-lengths in standard air to wave-lengths in vacuum and vice versa, the use of the dispersion formula adopted by the Joint Commission for Spectroscopy in Rome in I952, and given on p. 202 of this volume.

The question of accepting the combination principle as a basis for deriving secondary standards of wave-length was discussed. Meggers considered it a forward step to accept this method and noted that it would increase appreciably the available number of standards. Edlén reported on the calculations of $\mathrm{Fe} I$ lines, mentioned on p. 203 of this volume, and presented the final results in the form of two tables, one (Table $\mathbf{r}$ ) giving the energy levels and the other (Table 2) the wave-lengths calculated from these levels. The final calculation of Fe I levels is based on the measured wave-lengths of 575 
lines in the range of $9372 \AA$ to 25 or $\AA$, each wave-length being a weighted mean of all available determinations. Weights were assigned to the different wave-lengths according to the number and accuracy of determinations and to the wave-length region, the weights being inversely proportional to the square of the estimated wave-number uncertainty. The weights ranged from 2 to 60 on an arbitrary scale. A total of 55 even and 138 odd levels was involved. A number of additional levels were afterwards added, some of which were derived from one combination only. In the deep red and infra-red, where no observations with the standard long arc exist, only 'stable' lines had been utilized. In other parts of the spectrum some multiplets involving the high even terms $\mathrm{e}^{7} \mathrm{D}, \mathrm{e}^{5} \mathrm{D}$, and $e^{5} \mathrm{~F}$ had been included. Wave-lengths derived from combinations with high even levels are marked ' $\mathrm{e}$ ' in Table 2 to indicate that they are less stable than the others, and are recommendable as standards only when the standard long arc is being used. The wavelength table gives the calculated wave-lengths of ror 6 lines between $2080 \AA$ and I2,000 $\AA$. (The level combination for each line can be found in the monograph by H. N. Russell and C. E. Moore, Trans. Am. Phil. Soc. 34, part 2, 1944.)

A detailed account of the work will be published. Mimeographed copies of the tables may be obtained from the Department of Physics, University of Lund. The Commission passed a resolution recommending that the I.A.U. adopt as secondary standards of wavelength the calculated wave-lengths of ror6 lines in the spectrum of the iron arc in air, as given in the list presented at the Commission meeting, and included as Table 2 in this Report, p. 220. This list is to replace the iron standards previously adopted.

TABLE I. Fe I energy levels; arc-in-air values

\begin{tabular}{|c|c|}
\hline $\begin{array}{l}{ }^{5} \mathrm{D}_{4} \\
{ }^{5} \mathrm{D}_{3} \\
{ }^{5} \mathrm{D}_{2} \\
{ }^{5} \mathrm{D}_{1} \\
{ }^{5} \mathrm{D}_{0}\end{array}$ & $\begin{array}{r}0.000 \\
415 \cdot 932 \\
704 \cdot 003 \\
888 \cdot 132 \\
978 \cdot 076\end{array}$ \\
\hline & $\begin{array}{cc}6 & 928 \cdot 266 \\
7 & 376 \cdot 762 \\
7 & 728 \cdot 058 \\
7 & 985 \cdot 783 \\
8 & 154 \cdot 713\end{array}$ \\
\hline & $\begin{array}{l}11976 \cdot 239 \\
12560 \cdot 933 \\
12968 \cdot 552\end{array}$ \\
\hline a ${ }^{5} P_{3}$ & $\begin{array}{l}17550 \cdot 180 \\
17726 \cdot 985 \\
17927 \cdot 378\end{array}$ \\
\hline $\mathrm{a}{ }^{3} \mathrm{P}_{2}$ & $\begin{array}{l}18378 \cdot 187 \\
19552 \cdot 474 \\
20 \quad 037 \cdot 815\end{array}$ \\
\hline & $\begin{array}{l}19390 \cdot 165 \\
19621 \cdot 004 \\
19788 \cdot 248\end{array}$ \\
\hline & $\begin{array}{ll}20 & 641 \cdot 110 \\
20 & 874 \cdot 482 \\
21 & 038 \cdot 987\end{array}$ \\
\hline $\mathrm{G}_{3}$ & $\begin{array}{l}21715 \cdot 735 \\
21999 \cdot 132 \\
22 \quad 249 \cdot 430\end{array}$ \\
\hline & $\begin{array}{ll}22 & 838 \cdot 320 \\
22 & 946 \cdot 810 \\
23 & 051 \cdot 744\end{array}$ \\
\hline b ${ }^{3} \mathrm{G}_{5}$ & $\begin{array}{ll}23 & 783 \cdot 617 \\
24 & 118 \cdot 815\end{array}$ \\
\hline
\end{tabular}

c ${ }^{3} \mathrm{P}_{2}$
${ }^{3} \mathrm{P}_{1}$
a ${ }^{1} \mathrm{G}_{4}$
b ${ }^{3} \mathrm{H}_{6}$
${ }^{3} \mathrm{H}_{5}$
${ }^{3} \mathrm{H}_{4}$
a ${ }^{3} \mathrm{D}_{3}$
${ }^{3} \mathrm{D}_{2}$
a ${ }^{1} \mathrm{D}_{2}$
a ${ }^{1} \mathrm{H}_{5}$
a ${ }^{1} \mathrm{I}_{6}$
c ${ }^{3} \mathrm{~F}_{4}$
${ }^{3} \mathrm{~F}_{3}$
${ }^{3} \mathrm{~F}_{2}$
e ${ }^{2} \mathrm{D}_{5}$
${ }^{7} \mathrm{D}_{4}$
${ }^{7} \mathrm{D}_{3}$
${ }^{7} \mathrm{D}_{2}$
${ }^{2} \mathrm{D}_{1}$
e ${ }^{5} \mathrm{D}_{4}$
${ }^{5} \mathrm{D}_{3}$
${ }^{5} \mathrm{D}_{2}$
${ }^{5} \mathrm{D}_{1}$
${ }^{5} \mathrm{D}_{0}$
e ${ }^{5} \mathrm{~F}_{5}$
${ }^{5} \mathrm{~F}_{4}$
${ }^{5} \mathrm{~F}_{3}$
2 ${ }^{7} \mathrm{D}_{5}$
${ }^{7} \mathrm{D}_{4}$
${ }^{7} \mathrm{D}_{3}$
${ }^{2} \mathrm{D}_{2}$

$24335 \cdot 764$
$24772 \cdot 019$
$24574 \cdot 655$
$26 \quad 105 \cdot 908$
$26351 \cdot 049$
$26627 \cdot 612$
$26224 \cdot 961$
$26 \quad 623 \cdot 726$
$28604 \cdot 610$
$28819 \cdot 966$
$29313 \cdot 008$
$32873 \cdot 623$
$33412 \cdot 706$
$33765 \cdot 291$
$42815 \cdot 814$
$43163 \cdot 282$
$43434 \cdot 585$
$43633 \cdot 491$
$43763 \cdot 938$
$44676 \cdot 963$
$45061 \cdot 287$
$45333 \cdot 834$
$45509 \cdot 112$
$45595 \cdot 046$
$47005 \cdot 455$
$47377 \cdot 909$
$47755 \cdot 485$
$19350 \cdot 882$
$19562 \cdot 435$
$19757 \cdot 020$
$19912 \cdot 486$

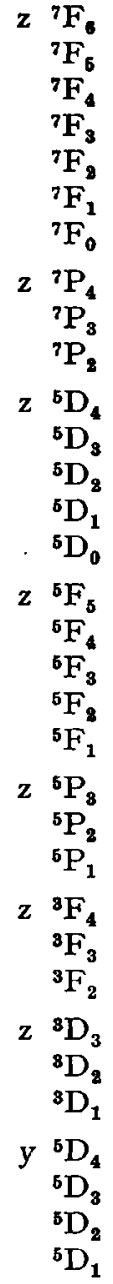

y ${ }^{5} \mathrm{~F}$

${ }^{5} \mathrm{~F}$

${ }^{6} \mathrm{~F}_{3}$

${ }^{3} \mathrm{~F}_{2}$

${ }^{5} \mathrm{~F}_{1}$

$23244 \cdot 826$

$23270 \cdot 374$

$23711 \cdot 443$

$24 \quad 180 \cdot 848$

24 506.902

25899.974

26 140 164

$26339 \cdot 682$

26 479.366

$26550 \cdot 466$

26 874.534

27 166.804

$27394 \cdot 676$

27 559.568

27 666.334

29 056.312

$29469 \cdot 010$

29732.721

$31307 \cdot 235$

31 805.059

$32 \quad 133.977$

31322.603

31 686.342

31 937.308

33 095.922

33 507.102

$33801 \cdot 555$

34017.084

${ }^{3} \mathrm{~F}_{2}$

y ${ }^{5} \mathrm{P}_{3}$

${ }^{5} \mathrm{P}_{2}$
33 695.378

34 039.495

34 328.733

34 547-192

34 692-130

33 946.920

$34362 \cdot 860$

$34555 \cdot 586$

34782.406

35 257.309

$35611 \cdot 608$

$35856 \cdot 386$

35 379-189

$35767 \cdot 546$

36 079.353

$\mathrm{y}^{8} \mathrm{~F}_{4} \quad 36686 \cdot 159$

$37162 \cdot 729$

37 521 144

36766.950

37 157.546

$37 \mathbf{4 0 9} \cdot \mathbf{5 3 0}$

y ${ }^{3} \mathrm{D}_{3} \quad 38 \mathrm{175} \cdot 332$

${ }^{3} \mathrm{D}_{2} \quad 38678.015$

${ }^{3} \mathrm{D}_{1} \quad 38995 \cdot 717$

$x{ }^{5} D_{4} \quad 39625 \cdot 784$

${ }^{5} \mathrm{D}_{3} \quad 39969 \cdot 830$

${ }^{\circ} \mathrm{D}_{2} \quad 40231 \cdot 312$

${ }^{5} \mathrm{D}_{1} \quad 40404 \cdot 492$

${ }^{5} \mathrm{D}_{0} \quad 40491 \cdot 260$

$x^{6} F_{5} \quad 40257 \cdot 300$

${ }^{5} \mathrm{~F}_{4} \quad 40594.413$

${ }^{6} \mathrm{~F}_{3} \quad 40842 \cdot 130$ 
TABLE I. Fe I energy levels (cont.)

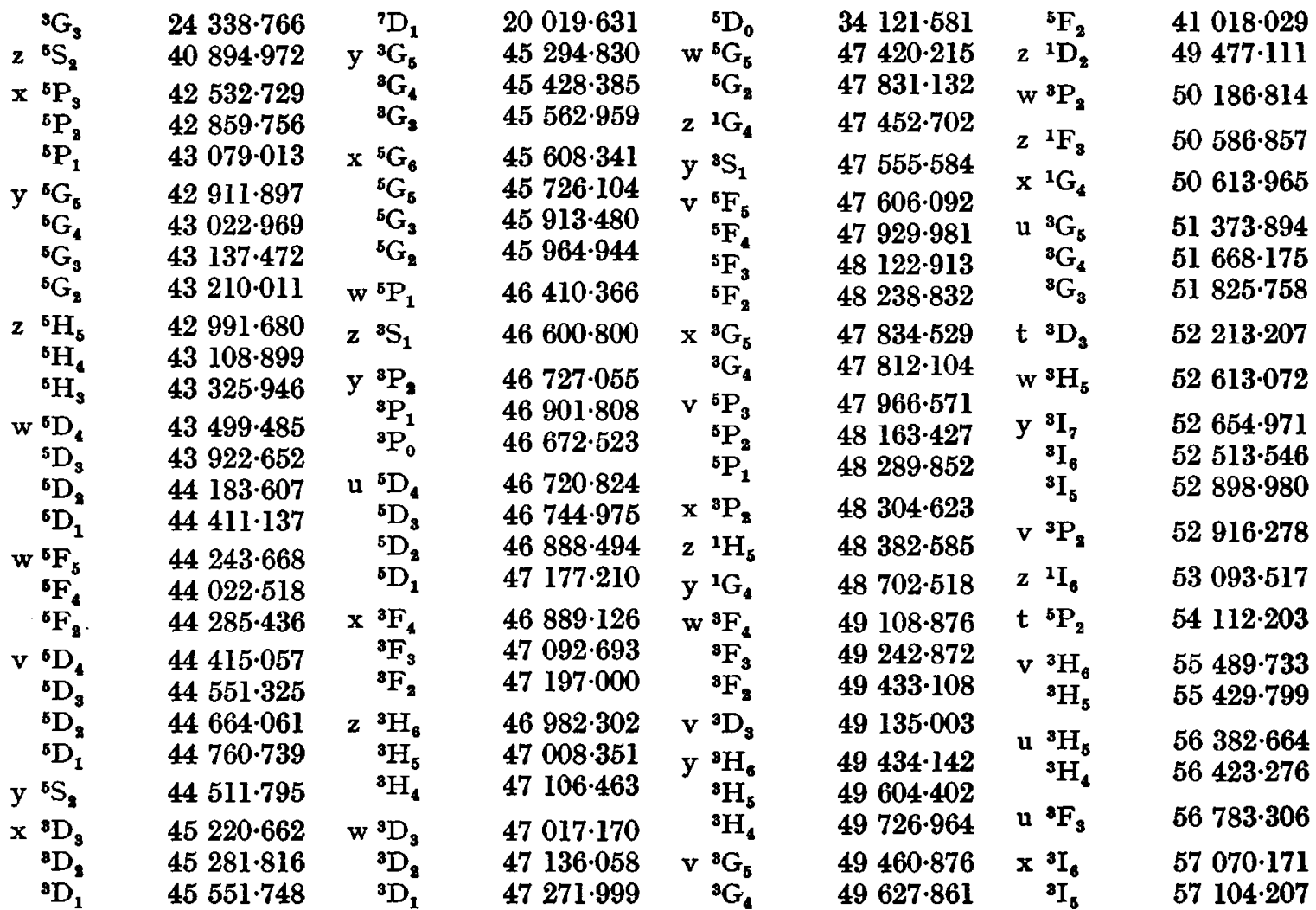

TABLE 2. Wave-lengths in the iron arc in air
11973.067
$11884 \cdot 097$
$11882 \cdot 861$
$11783 \cdot 275$
$11689 \cdot 988$
$11638 \cdot 279$
$11607 \cdot 587$
$11593 \cdot 600$
$11439 \cdot 129$
$11422 \cdot 335$
$11374 \cdot 095$
$11119 \cdot 809$
$10395 \cdot 811$
$10340 \cdot 898$
9372.904
9362-371
$9359 \cdot 419$
$9210 \cdot 033$
$9146 \cdot 138$
$9118 \cdot 892$
$9089 \cdot 415$
9088.324
8999.564
$8975 \cdot 410$
8943.078
$8868 \cdot 442$

\begin{tabular}{|c|c|}
\hline $8838 \cdot 434$ & 7443.019 \\
\hline $8824 \cdot 227$ & $7418 \cdot 673$ \\
\hline $8804 \cdot 637$ & $7401 \cdot 690$ \\
\hline $8757 \cdot 192$ & $7284 \cdot 842$ \\
\hline $8688 \cdot 632$ & $7223 \cdot 668$ \\
\hline $8674 \cdot 753$ & $7219 \cdot 686$ \\
\hline $8661 \cdot 907$ & $7207 \cdot 116$ \\
\hline $8621 \cdot 613$ & 7132.990 \\
\hline $8611 \cdot 807$ & $7112 \cdot 177$ \\
\hline $8582 \cdot 268$ & $7107 \cdot 462$ \\
\hline $8515 \cdot 122$ & $7068 \cdot 413$ \\
\hline $8514 \cdot 079$ & $7024 \cdot 068$ \\
\hline $8468 \cdot 414$ & $7016 \cdot 062$ \\
\hline $8387 \cdot 780$ & 6988.531 \\
\hline $8365 \cdot 642$ & $6978 \cdot 856$ \\
\hline $8327 \cdot 063$ & $6945 \cdot 208$ \\
\hline 8293.523 & $6839 \cdot 837$ \\
\hline $8239 \cdot 130$ & $6806 \cdot 851$ \\
\hline $8096 \cdot 881$ & $6750 \cdot 15$ \\
\hline $8075 \cdot 156$ & $6703 \cdot 574$ \\
\hline $8047 \cdot 621$ & $6677 \cdot 99$ \\
\hline $7912 \cdot 867$ & $6663 \cdot 444$ \\
\hline $7748 \cdot 278$ & $6609 \cdot 117$ \\
\hline $7723 \cdot 213$ & $6593 \cdot 87$ \\
\hline $7664 \cdot 301$ & $6592 \cdot 92$ \\
\hline $7583 \cdot 797$ & $6575 \cdot 024$ \\
\hline
\end{tabular}

6574.231

6546.245

6518.374

6498.943

6494.985

$6481 \cdot 877$

6475.632

6462.730

$6430 \cdot 852$

$6421 \cdot 355$

$6411 \cdot 659 \mathrm{e}$

$6408.028 \mathrm{e}$

$6400 \cdot 321$

$6400.013 \mathrm{e}$

$6393 \cdot 605$

$6380 \cdot 746$

6358.696

$6355 \cdot 035$

6344.154

$6336.833 \mathrm{e}$

6335.337

6322.691

6318.023

$6301.510 \mathrm{e}$

6297.798

$6280 \cdot 621$ 
TABLE 2. Wave-lengths in the iron arc in air (cont.)

\begin{tabular}{|c|c|c|c|}
\hline $6265 \cdot 140$ & $5269 \cdot 5402$ & $4910 \cdot 0253 \mathrm{e}$ & $4442 \cdot 3428$ \\
\hline $6256 \cdot 367$ & $5266.5626 \mathrm{e}$ & $4903 \cdot 3169 \mathrm{e}$ & $4439 \cdot 8849$ \\
\hline $6254 \cdot 263$ & $5263.3134 \mathrm{e}$ & $4891 \cdot 4989 \mathrm{e}$ & $4436 \cdot 9246$ \\
\hline $6252 \cdot 561$ & $5250 \cdot 6490$ & $4890 \cdot 7616 \mathrm{e}$ & $4435 \cdot 1500$ \\
\hline $6246.329 \mathrm{e}$ & $5242 \cdot 4955$ & $4878 \cdot 2182 \mathrm{e}$ & $4432 \cdot 5729$ \\
\hline $6240 \cdot 649$ & $5232.9474 \mathrm{e}$ & $4872 \cdot 1444 \mathrm{e}$ & $4430 \cdot 6175$ \\
\hline $6232 \cdot 650 \mathrm{e}$ & $5227 \cdot 1911$ & $4871 \cdot 3244 \mathrm{e}$ & $4427 \cdot 3118$ \\
\hline $6230 \cdot 728$ & $5226 \cdot 8686 \mathrm{e}$ & $4859 \cdot 7480 \mathrm{e}$ & 4422.5703 \\
\hline $6219 \cdot 286$ & $5217.3964 \mathrm{e}$ & $4855 \cdot 6812 \mathrm{e}$ & $4415 \cdot 1250$ \\
\hline $6213 \cdot 435$ & $5216 \cdot 2770$ & $4844 \cdot 0158$ & $4408 \cdot 4176$ \\
\hline $6200 \cdot 319$ & $5215 \cdot 1871 \mathrm{e}$ & $4839 \cdot 5510$ & $4407 \cdot 7130$ \\
\hline $6191 \cdot 563$ & $5208 \cdot 6007 \mathrm{e}$ & $4789 \cdot 6537$ & $4404 \cdot 7525$ \\
\hline $6173 \cdot 341$ & $5204 \cdot 5840$ & $4788 \cdot 7606$ & $4401 \cdot 4456$ \\
\hline $6165 \cdot 364$ & $5202 \cdot 3395$ & $\mathbf{4 7 8 6} \cdot 8106$ & $4390 \cdot 9542$ \\
\hline $6157 \cdot 732$ & $5198 \cdot 7149$ & $4771 \cdot 7008$ & $4389 \cdot 2467$ \\
\hline $6151 \cdot 623$ & 5194.9441 & $4741 \cdot 5321$ & $4387 \cdot 8959$ \\
\hline $6141 \cdot 741 \mathrm{e}$ & $5192 \cdot 3509 \mathrm{e}$ & $4740 \cdot 3434$ & $4383 \cdot 5473$ \\
\hline $6137 \cdot 697$ & $5191 \cdot 4615 \mathrm{e}$ & $4736 \cdot 7807 \mathrm{e}$ & $4375 \cdot 9318$ \\
\hline 6137.000 & $5171 \cdot 5987$ & $4733 \cdot 5955$ & $4369 \cdot 7745$ \\
\hline $6136 \cdot 621$ & 5168.9003 & $4710 \cdot 2864$ & 4367.9059 \\
\hline $6065 \cdot 488$ & $5167 \cdot 4905$ & $4707 \cdot 4908$ & $4367 \cdot 5811$ \\
\hline $6027 \cdot 057$ & 5166.2841 & $4707 \cdot 2807 \mathrm{e}$ & $4358 \cdot 5037$ \\
\hline $5956 \cdot 697$ & 5151.9143 & $4691 \cdot 4144$ & $4352 \cdot 7371$ \\
\hline $5916 \cdot 252$ & $5150 \cdot 8425$ & $4683 \cdot 5640$ & $4351 \cdot 5465$ \\
\hline $5709 \cdot 3864 \mathrm{e}$ & 5142.9320 & $4680 \cdot 2983$ & $4348 \cdot 9398$ \\
\hline $5701 \cdot 5511$ & $5141 \cdot 7424$ & $4668 \cdot 1422 \mathrm{e}$ & $4346 \cdot 5571$ \\
\hline $5658 \cdot 8247 \mathrm{e}$ & $5139 \cdot 4702 \mathrm{e}$ & 4661.9726 & $4337 \cdot 0484$ \\
\hline $5624 \cdot 5501 \mathrm{e}$ & $5139 \cdot 2578 \mathrm{e}$ & $4654 \cdot 5020$ & $4326 \cdot 7555$ \\
\hline $5615 \cdot 6521 \mathrm{e}$ & $5131 \cdot 4734$ & $4647 \cdot 4370$ & $4325 \cdot 7647$ \\
\hline $5615 \cdot 3039$ & $5127 \cdot 3624$ & 4632.9149 & $4315 \cdot 0872$ \\
\hline $5602.9529 \mathrm{e}$ & $5123 \cdot 7231$ & $4630 \cdot 1248$ & $4309 \cdot 3771$ \\
\hline $5586 \cdot 7634 \mathrm{e}$ & $5110 \cdot 4139$ & $4625.0527 \mathrm{e}$ & $4307 \cdot 9048$ \\
\hline $5572 \cdot 8501 \mathrm{e}$ & $5107 \cdot 6439$ & $4618 \cdot 7604$ & $4305 \cdot 4545$ \\
\hline $5569 \cdot 6256 \mathrm{e}$ & $5107 \cdot 4505$ & $4602 \cdot 9446$ & $4304 \cdot 5436$ \\
\hline $5506 \cdot 7824$ & $5098 \cdot 7030$ & $4602 \cdot 0040$ & $4302 \cdot 1882$ \\
\hline $5501 \cdot 4686$ & $5083 \cdot 3413$ & $4595 \cdot 3627$ & $4299 \cdot 2409 \mathrm{e}$ \\
\hline $5497 \cdot 5196$ & $5079 \cdot 7426$ & $4592 \cdot 6547$ & $4298 \cdot 0403$ \\
\hline $5455 \cdot 6131$ & $5079 \cdot 2279$ & $4574 \cdot 7225$ & $4294 \cdot 1271$ \\
\hline 5446.9197 & $5068 \cdot 7730 \mathrm{e}$ & $4547 \cdot 8505$ & $4288 \cdot 1484$ \\
\hline $5434 \cdot 5268$ & $5051 \cdot 6379$ & $4547 \cdot 0206$ & $4285 \cdot 4453$ \\
\hline $5429 \cdot 6999$ & $5049 \cdot 8253$ & $4531 \cdot 1520$ & $4282 \cdot 4057$ \\
\hline $5405 \cdot 7781$ & $5041 \cdot 7585$ & $4528 \cdot 6175$ & $4271 \cdot 7634$ \\
\hline $5397 \cdot 1311$ & $5041 \cdot 0747$ & $4517 \cdot 5289$ & $427 \mathrm{l} \cdot 1589 \mathrm{e}$ \\
\hline $5393 \cdot 1752 \mathrm{e}$ & $5028 \cdot 1331$ & $4514 \cdot 1876$ & $4266 \cdot 9675$ \\
\hline $5371 \cdot 4926$ & $5012 \cdot 0712$ & $4494 \cdot 5669$ & $4260.4794 \mathrm{e}$ \\
\hline $5365 \cdot 4062$ & $5006 \cdot 1254 \mathrm{e}$ & $4490 \cdot 0872$ & $4258 \cdot 3174$ \\
\hline $5341 \cdot 0255$ & $5002 \cdot 7998 \mathrm{e}$ & $4489 \cdot 7416$ & $4250 \cdot 7896$ \\
\hline $5339.9371 \mathrm{e}$ & $4994 \cdot 1323$ & $4482 \cdot 2563$ & $4250 \cdot 1248 \mathrm{e}$ \\
\hline $5332 \cdot 9020$ & $4985 \cdot 5539 \mathrm{e}$ & $4482 \cdot 1720$ & $4248 \cdot 2275$ \\
\hline $5328 \cdot 5336$ & $4966.0968 \mathrm{e}$ & $4480 \cdot 1397$ & $4245 \cdot 2594$ \\
\hline $5328 \cdot 0418$ & $4957 \cdot 6059 \mathrm{e}$ & $4476 \cdot 0206$ & $4235 \cdot 9433 \mathrm{e}$ \\
\hline $5324 \cdot 1864$ e & $4957 \cdot 3054 \mathrm{e}$ & $4466 \cdot 5542$ & $4233.6089 \mathrm{e}$ \\
\hline $5322 \cdot 0456$ & $4950 \cdot 1144 \mathrm{e}$ & $4464 \cdot 7691$ & $4229 \cdot 7561$ \\
\hline $5307 \cdot 3633$ & $4946 \cdot 3944 \mathrm{e}$ & $4461 \cdot 6544$ & $4226 \cdot 4263$ \\
\hline $5302 \cdot 3073 \mathrm{e}$ & $4939 \cdot 6896$ & $4459 \cdot 1213$ & $4222 \cdot 2181 \mathrm{e}$ \\
\hline $5283 \cdot 6283 \mathrm{e}$ & $4938.8206 \mathrm{e}$ & $4456 \cdot 3294$ & $4219 \cdot 364 I$ \\
\hline $5281 \cdot 7970 \mathrm{e}$ & $4924 \cdot 7753$ & $4454 \cdot 3835$ & $4216 \cdot 1854$ \\
\hline $5273 \cdot 1708 \mathrm{e}$ & $4920 \cdot 5096 \mathrm{e}$ & $4447 \cdot 7212$ & $4210.3497 \mathrm{e}$ \\
\hline $5270 \cdot 3602$ & $4919 \cdot 0003 \mathrm{e}$ & $4443 \cdot 1963$ & $4207 \cdot 1298$ \\
\hline
\end{tabular}


TABLE 2. Wave-lengths in the iron arc in air (cont.)

$4206 \cdot 6985$

4203.9867

4202.0320

4199.0981

$4198 \cdot 3098 \mathrm{e}$

4195.6205

$4191 \cdot 4358 \mathrm{e}$

$4187.8015 \mathrm{e}$

$4187.0436 \mathrm{e}$

4184.8941

$4182 \cdot 3846$

$4181 \cdot 7571$

4177.5949

$4175 \cdot 6386$

4174.9137

4173.9230

4173.3178

$4172 \cdot 7454$

4170.9044

4156.8021

4154-5021

4152-1704

4147.6719

4143.8703

4143.4174

4139.9288

$4134 \cdot 6798$

4132.9024

4132.0603

$4127 \cdot 6113$

$4125 \cdot 8831$

$4121 \cdot 8050$

4120.2087

4118.5484

$4114 \cdot 4485$

4109-8053

$\mathbf{4 1 0 7 \cdot 4 9 1 7}$

4100.7389

4095.9731

$4091 \cdot 5566$

$4079 \cdot 8411$

$4078 \cdot 3563$

4074. 7889

4071.7399

$4067 \cdot 2738$

4063.5963

$4062 \cdot 4440$

$4058 \cdot 7562$

4057-3456

4055.0376

4045.8147

4044.6125

4032.6294

$4030 \cdot 1855$

$4021 \cdot 8696$

$4017 \cdot 1524$

4009.7154

$4007 \cdot 2735$

4005.2440
$4001 \cdot 6627$

$4000 \cdot 4598$

3998.0554

3997-3952

3995.9861

3994. 1166

3990-3766

3983.9593

3981.7743

$3977 \cdot 7437$

$3971 \cdot 3250$

$3969 \cdot 2595$

3967.4234

3966.0645

3964.5173

$3956 \cdot 6796$

3956.4574

3953 1548

3952.6045

3949.9558

3948.7778

3944.8924

$3943 \cdot 3414$

$3942 \cdot 4418$

$3940 \cdot 8797$.

3937.3310

$\mathbf{3 9 3 5} \cdot 8143$

$3930 \cdot 2981$

3927.9216

$3925 \cdot 6460$

3922.9134

$3920 \cdot 2601$

$3919 \cdot 0681$

3918.3174

$3917 \cdot 1834$

3913.6339

$3910 \cdot 8461$

3907.9371

3906.4814

3903.9011

3902.9484

$3899 \cdot 7086$

3898.0111

$3897 \cdot 4515$

$3895 \cdot 6579$

3893.9141

3893.3935

3888.5165

$3887 \cdot 0504$

3886.2839

3885.5121

3884.3609

3878.5745

3878.0206

3876.0414

3873.7624

3872.5032

$3871 \cdot 7513$

3869.5615
$3867 \cdot 2184$

3865.5256

3859.9132

3859.2143

3856.3731

3852.5752

$3850 \cdot 8193$

3849.9694

3846.8023

$3845 \cdot 1706$

$3843 \cdot 2596$

3841.0499

$3840 \cdot 4397$

$3839 \cdot 2584$

$3837 \cdot 1370$

3834.2244

3833.3103

3830.8638

$3830 \cdot 7607$

$\mathbf{3 8 2 9} \cdot 7637$

$3827 \cdot 8256$

$3827 \cdot 5746$

3825.8834

$3824 \cdot 4455$

3824.0763

$3821 \cdot 8357$

3821-1807

$3820 \cdot 4274$

3816.3421

$\mathbf{3 8 1 5} \cdot 8430$

3814.5247

3812.9658

3808.7306

3807.5392

3806.6992

3805.3450

$3801 \cdot 6817$

$3799 \cdot 5498$

3798.5134

3797.9502

$3795 \cdot 0045$

$3790 \cdot 0943$

$3789 \cdot 1783$

3787-8825

3786.6781

$3778 \cdot 6986$

$3777 \cdot 4521$

$3776 \cdot 4553$

3774.8266

$3767 \cdot 1939$

3765.5414

3763.7910

$3761 \cdot 4103$

$3760 \cdot 5335$

$\mathbf{3 7 5 8 \cdot 2 3 5 0}$

3753.6134

$3749 \cdot 4875$

$3748 \cdot 2639$

$\mathbf{3 7 4 5} .9013$
3745.5623

$\mathbf{3 7 4 3 \cdot 3 6 4 0}$

3738.3078

3737-1333

$3734 \cdot 8659$

3733-3191

3731.3761

$\mathbf{3 7 3 0} \cdot 3884$

3728.6696

$3727 \cdot 6211$

$\mathbf{3 7 2 4} \cdot \mathbf{3 7 9 6}$

$3722 \cdot 5642$

$3722 \cdot 0263$

3719.9367

3718.4092

3715.9136

3711-2243

3709.2484

$\mathbf{3 7 0 7 . 9 2 1 6}$

$\mathbf{3 7 0 7 \cdot 8 2 3 1}$

$3705 \cdot 5674$

$\mathbf{3 7 0 4} \cdot 4635$

$\mathbf{3 6 8 7} \cdot 4589$

3687.0982

$3684 \cdot 1102$

3683.0562

3679.9152

$\mathbf{3 6 7 8} \cdot 8620$

3677-6309

3676.3135

$3669 \cdot 5229$

$\mathbf{3 6 5 9 . 5 1 8 8}$

$3655 \cdot 4671$

3651-4699

$3650 \cdot 2811$

$3649 \cdot 5090$

$3649 \cdot 3045$

$\mathbf{3 6 4 7} \cdot 8439$

$3640 \cdot 3918$

3638.2998

3632.9799

$3631 \cdot 4646$

$3623 \cdot 1878$

$3621 \cdot 4640$

$\mathbf{3 6 1 8 \cdot 7 6 9 4}$

3608.8609

$3606 \cdot 6821$

3603.2068

3589-1063

3586.9861

3585.7068

3585-3206

3584.6627

3581-6499

3571-2265

3570.0996

3568.9778

3565.3807

3558.5170 
TABLE 2. Wave-lengths in the iron arc in air (cont.)

\begin{tabular}{|c|c|}
\hline $3554 \cdot 1196$ & 3355.2287 \\
\hline $3540 \cdot 7111$ & $3351 \cdot 7457$ \\
\hline $3526 \cdot 4695$ & $3351 \cdot 5239$ \\
\hline $3526 \cdot 1676$ & $3347 \cdot 9271$ \\
\hline $3526 \cdot 0415$ & $3342 \cdot 2163$ \\
\hline $3524 \cdot 2417$ & $3340 \cdot 5666$ \\
\hline $3521 \cdot 2630$ & $3337 \cdot 6664$ \\
\hline $3513 \cdot 8196$ & $3335 \cdot 7699$ \\
\hline $3506 \cdot 5004$ & $3334 \cdot 2201$ \\
\hline $3504 \cdot 8636$ & $3331 \cdot 6133$ \\
\hline $3500 \cdot 5675$ & $3328 \cdot 8667$ \\
\hline $3497 \cdot 8420$ & $3327 \cdot 4970$ \\
\hline $3495 \cdot 2879$ & $\mathbf{3 3 2 4} \cdot 5385$ \\
\hline $3490 \cdot 5749$ & $\mathbf{3 3 2 3 \cdot 7 3 7 5}$ \\
\hline $3485 \cdot 3418$ & $3314 \cdot 7420$ \\
\hline $3483 \cdot 0090$ & $3305 \cdot 9719$ \\
\hline $3476 \cdot 7036$ & 3298-1331 \\
\hline $3475 \cdot 4511$ & $3292 \cdot 5910$ \\
\hline $3471 \cdot 3460$ & 3290.9899 \\
\hline $3471 \cdot 2672$ & $3286 \cdot 7541$ \\
\hline $3468 \cdot 8474$ & 3284-5888 \\
\hline $3466 \cdot 5001$ & $3280 \cdot 2613$ \\
\hline $3465 \cdot 8621$ & 3276.4713 \\
\hline $3463 \cdot 3044$ & $3271 \cdot 6842$ \\
\hline $3462 \cdot 3539$ & $3271 \cdot 0014$ \\
\hline $3452 \cdot 2760$ & $3265 \cdot 6182$ \\
\hline 3451.9166 & 3265.0473 \\
\hline $3450 \cdot 3304$ & $3263 \cdot 3697$ \\
\hline $3447 \cdot 2797$ & $3257 \cdot 5940$ \\
\hline $3445 \cdot 1508$ & $3254 \cdot 3628$ \\
\hline 3443.8775 & 3252.9160 \\
\hline $3442 \cdot 6709$ & $3250 \cdot 6250$ \\
\hline 3440.9899 & 3246.4816 \\
\hline $3440 \cdot 6069$ & $3246 \cdot 0054$ \\
\hline $3428 \cdot 1948$ & $3236 \cdot 2231$ \\
\hline $3427 \cdot 1213$ & $3234 \cdot 6138$ \\
\hline $3424 \cdot 2861$ & $3229 \cdot 1221$ \\
\hline $3422 \cdot 6583$ & $3226 \cdot 7137$ \\
\hline $3417 \cdot 8428$ & $3214: 3964$ \\
\hline $3415 \cdot 5318$ & $3200 \cdot 7854$ \\
\hline $3413 \cdot 1339$ & $3193 \cdot 2268$ \\
\hline $3407 \cdot 4611$ & $3191 \cdot 6599$ \\
\hline $3406 \cdot 8021$ & $3184 \cdot 8955$ \\
\hline $3404 \cdot 3557$ & 3182.9798 \\
\hline 3401.5200 & $3180 \cdot 7562$ \\
\hline $3399 \cdot 3356$ & $3161 \cdot 3728$ \\
\hline $3397 \cdot 6403$ & $3148 \cdot 4078$ \\
\hline 3396.9774 & $3143 \cdot 2434$ \\
\hline $3394 \cdot 5854$ & $3142 \cdot 8908$ \\
\hline $3392 \cdot 6540$ & $3134 \cdot 1115$ \\
\hline $3392 \cdot 3058$ & $\mathbf{3 1 2 9} 3349$ \\
\hline $\mathbf{3 3 8 3} .9808$ & $3120 \cdot 4364$ \\
\hline $3382 \cdot 4042$ & $3119 \cdot 4956$ \\
\hline $3380 \cdot 1117$ & $3116 \cdot 6337$ \\
\hline $3379 \cdot 0206$ & $3100 \cdot 6667$ \\
\hline $3372 \cdot 0744$ & $3100 \cdot 3054$ \\
\hline $3370 \cdot 7852$ & 3099.9695 \\
\hline $3359 \cdot 4876$ & $3099 \cdot 8968$ \\
\hline $3356 \cdot 4030$ & $3093 \cdot 8063$ \\
\hline
\end{tabular}

$3091.5786 \quad 2936.9049$

$\mathbf{3 0 8 3 \cdot 7 4 3 0} \quad \mathbf{2 9 2 9 \cdot 6 1 9 5}$

$3075 \cdot 7214 \quad 2929 \cdot 0085$

3068.1749 $2925 \cdot 9012$

3067.2457 $2920 \cdot 6915$

$3067 \cdot 1196 \quad 2914 \cdot 3055$

$3060 \cdot 9849 \quad 2912 \cdot 2582$

$\begin{array}{ll}3059 \cdot 0871 & \mathbf{2 9 1 2} \cdot 1589\end{array}$

$3057 \cdot 4471 \quad 2901 \cdot 3820$

$\begin{array}{ll}3055 \cdot 2638 & 2895 \cdot 0362\end{array}$

$3047 \cdot 6060 \quad 2894 \cdot 5055$

$3042 \cdot 6667 \quad \mathbf{2 8 9 3 \cdot 8 8 2 2}$

$3042 \cdot 0215 \quad \mathbf{2 8 8 6 \cdot 3 1 7 4}$

$3041 \cdot 7401 \quad 2880.5806$

$3041 \cdot 6386 \quad 2877 \cdot 3021$

$3040 \cdot 4281$
$2875 \cdot 3034$

$3037 \cdot 7809 \quad 2874 \cdot 1733$

$3037 \cdot 3901 \quad \mathbf{2 8 7 2 \cdot 3 3 4 6}$

$3030 \cdot 1494 \quad 2869 \cdot 3083$

$3029 \cdot 2351 \quad 2867 \cdot 5632$

$3026 \cdot 4637 \quad 2866 \cdot 6264$

3025.8442 $2863 \cdot 8644$

$3024.0337 \quad 2863.4311$

$3021 \cdot 0743 \quad 2862 \cdot 4952$

$3020 \cdot 6405 \quad 2858 \cdot 8970$

$3020 \cdot 4918 \quad 2853 \cdot 6855$

3018.9848
$2851 \cdot 7979$

$3017 \cdot 6288 \quad 2848 \cdot 7153$

3014.1747 $2846 \cdot 8312$

$3009 \cdot 5707 \quad 2845.5959$

3009.0945 2843.9775

3008.1399 $2843 \cdot 6314$

$\begin{array}{ll}3007 \cdot 2832 & 2840 \cdot 9382\end{array}$

$\begin{array}{ll}3007 \cdot 1469 & 2840 \cdot 4229\end{array}$

$3003 \cdot 0323 \quad 2838 \cdot 1205$

$\begin{array}{ll}3000 \cdot 9489 & 2835 \cdot 9511\end{array}$

$3000 \cdot 4527 \quad 2835 \cdot 4574$

$2999 \cdot 5125 \quad 2832 \cdot 4364$

$2996 \cdot 3864 \quad \mathbf{2 8 2 8} \cdot 8094$

$2994.5033 \quad 2827.8931$

$2994 \cdot 4281$
$2825 \cdot 6888$

$2990 \cdot 3933 \quad 2825 \cdot 5569$

$\mathbf{2 9 8 8 \cdot 4 7 3 0} \mathbf{2 8 2 3 \cdot 2 7 6 7}$

$2987.2923 \quad 2820 \cdot 8039$

$2986 \cdot 4569$
$2817 \cdot 5047$

$2983 \cdot 5714 \quad 2813 \cdot 2877$

$2981 \cdot 4459 \quad 2808 \cdot 3281$

$2973 \cdot 2368 \quad 2807 \cdot 2461$

2973.1336 2806.9852

$2969 \cdot 4759 \quad 2804 \cdot 5212$

$2969 \cdot 3606 \quad 2803 \cdot 1675$

$2966 \cdot 8997 \quad 2797 \cdot 7765$

$2965 \cdot 2561 \quad 2796 \cdot 8721$

2959.9929
$2795 \cdot 5409$

$2957.3660 \quad 2795 \cdot 0065$

$2954 \cdot 6543 \quad 2794 \cdot 7033$

$2953.9411 \quad 2791 \cdot 7867$

$2947.8773 \quad 2787.9331$

$\begin{array}{ll}2941 \cdot 3438 & 2781 \cdot 8368\end{array}$ 
TABLE 2. Wave-lengths in the iron arc in air (cont.)

\begin{tabular}{|c|c|c|c|}
\hline $2778 \cdot 2214$ & $2673 \cdot 2142$ & 24965343 & $2259 \cdot 5109$ \\
\hline $2772 \cdot 1107$ & $2667 \cdot 9138$ & $2494 \cdot 2525$ & $2251 \cdot 8749$ \\
\hline $2772 \cdot 0748$ & $2666 \cdot 9665$ & $2491-1562$ & $2250 \cdot 7911$ \\
\hline $2769 \cdot 6717$ & $2666 \cdot 8133$ & $2490 \cdot 6454$ & $2245 \cdot 6536$ \\
\hline $2769 \cdot 2985$ & $2666 \cdot 3998$ & $2488 \cdot 1437$ & 2242.5725 \\
\hline $2767 \cdot 5232$ & $2662 \cdot 0574$ & $2487 \cdot 3705$ & $2231 \cdot 2138$ \\
\hline 2766.9104 & $2656 \cdot 7933$ & $2483 \cdot 2718$ & $2229 \cdot 0735$ \\
\hline $2762 \cdot 7732$ & $2651 \cdot 7075$ & $2479 \cdot 7774$ & $2228 \cdot 1722$ \\
\hline $2762 \cdot 0275$ & $2647 \cdot 5588$ & $2479 \cdot 4813$ & $2211 \cdot 2364$ \\
\hline $2761 \cdot 7810$ & $2645 \cdot 4230$ & $2474 \cdot 8151$ & $2210 \cdot 6894$ \\
\hline $2757 \cdot 3170$ & $2643 \cdot 9992$ & $2472 \cdot 8962$ & 2207.0692 \\
\hline $2756 \cdot 3295$ & $2641 \cdot 6468$ & $2468 \cdot 8803$ & $2196 \cdot 0427$ \\
\hline $2756 \cdot 2677$ & $2636 \cdot 4794$ & $2467 \cdot 7330$ & $2191 \cdot 2052$ \\
\hline $2754 \cdot 4273$ & $2635 \cdot 8100$ & $2465 \cdot 1500$ & $2187 \cdot 1950$ \\
\hline $2754 \cdot 0332$ & 2632.5951 & $2462 \cdot 6483$ & $2186 \cdot 8933$ \\
\hline $2750 \cdot 1415$ & $2632 \cdot 2382$ & $2462 \cdot 1822$ & $2180 \cdot 8692$ \\
\hline $2747 \cdot 5564$ & $2623 \cdot 3669$ & $2457 \cdot 5980$ & $2176 \cdot 8414$ \\
\hline $2744 \cdot 5287$ & $2618 \cdot 7111$ & $2453 \cdot 4767$ & $2173 \cdot 2146$ \\
\hline 2744.0691 & $2618 \cdot 0191$ & $2447 \cdot 7108$ & 2172.5858 \\
\hline $2742 \cdot 4064$ & $2614 \cdot 4952$ & $2445 \cdot 2134$ & $2171 \cdot 2976$ \\
\hline $2742 \cdot 2554$ & $2612 \cdot 7734$ & $2443 \cdot 8728$ & 2164.5495 \\
\hline $2742 \cdot 0164$ & $2606 \cdot 8280$ & $2438 \cdot 1831$ & $2163 \cdot 8633$ \\
\hline $2741 \cdot 5781$ & $2605 \cdot 6578$ & $2389 \cdot 9732$ & $2161 \cdot 5802$ \\
\hline $2738 \cdot 2143$ & $2584 \cdot 5370$ & $2381 \cdot 8356$ & $2159 \cdot 6583$ \\
\hline $2737 \cdot 3108$ & $2580 \cdot 4542$ & $2374 \cdot 5192$ & 2158.9207 \\
\hline $2735 \cdot 4762$ & $2580 \cdot 0662$ & $2373 \cdot 6250$ & $2157 \cdot 7951$ \\
\hline $2734 \cdot 6165$ & $2576 \cdot 6916$ & $2371 \cdot 4313$ & $2155 \cdot 0203$ \\
\hline $2734 \cdot 2691$ & $2564 \cdot 5609$ & $2369 \cdot 4567$ & $2153 \cdot 0075$ \\
\hline $2734 \cdot 0060$ & $2561 \cdot 8562$ & $2329 \cdot 6413$ & $2150 \cdot 1850$ \\
\hline $2733 \cdot 5816$ & $2560 \cdot 5576$ & $2320-3585$ & $2145 \cdot 1901$ \\
\hline $2730 \cdot 9822$ & $2556 \cdot 3043$ & $2313 \cdot 1048$ & $2141 \cdot 7188$ \\
\hline $2728 \cdot 9703$ & $2552 \cdot 8318$ & $2308 \cdot 9999$ & $2141 \cdot 0872$ \\
\hline $2728 \cdot 0212$ & $2549 \cdot 6142$ & $2303 \cdot 5815$ & $2139 \cdot 6987$ \\
\hline $2725 \cdot 6021$ & $2545 \cdot 9795$ & $2301 \cdot 6849$ & 2138.5932 \\
\hline $2724 \cdot 9542$ & $2540 \cdot 9734$ & $2299 \cdot 2209$ & $2132 \cdot 0177$ \\
\hline $2723 \cdot 5786$ & $2539 \cdot 3576$ & $2298 \cdot 1699$ & $2126 \cdot 2108$ \\
\hline $2720 \cdot 9035$ & $2537 \cdot 4598$ & $2297 \cdot 7877$ & $2115 \cdot 1697$ \\
\hline $2718 \cdot 4365$ & $2535 \cdot 6086$ & 2296.9279 & $2114 \cdot 6003$ \\
\hline $2717 \cdot 7874$ & $2532 \cdot 8764$ & $2293 \cdot 8482$ & 2112.9696 \\
\hline $2714 \cdot 8697$ & $2529 \cdot 8370$ & 2292.5249 & 2108.9598 \\
\hline $2711 \cdot 6560$ & $2529 \cdot 1361$ & $2291 \cdot 6274$ & $2108 \cdot 3027$ \\
\hline $2706 \cdot 5829$ & $2527 \cdot 4358$ & $2287 \cdot 2505$ & $2108 \cdot 1371$ \\
\hline $2699 \cdot 1075$ & $2524 \cdot 2939$ & $2284 \cdot 0864$ & $2106 \cdot 3953$ \\
\hline $2697 \cdot 0224$ & $2522 \cdot 8505$ & $2283 \cdot 6557$ & $2103 \cdot 0534$ \\
\hline $2695 \cdot 0357$ & $2521 \cdot 9197$ & $2283 \cdot 3045$ & $2102 \cdot 3542$ \\
\hline $2692 \cdot 2496$ & $2519 \cdot 6305$ & $2276 \cdot 0263$ & $2100 \cdot 7984$ \\
\hline $2690 \cdot 0694$ & $2518 \cdot 1029$ & 2272.0703 & $2100 \cdot 1464$ \\
\hline $2689 \cdot 8305$ & $2516 \cdot 5716$ & $2270 \cdot 8628$ & $2093 \cdot 6853$ \\
\hline $2689 \cdot 2130$ & $2510 \cdot 8362$ & $2269 \cdot 0990$ & $2090 \cdot 3837$ \\
\hline $2680 \cdot 4540$ & $2501 \cdot 6946$ & $2267 \cdot 0853$ & $2087 \cdot 5115$ \\
\hline $2679 \cdot 0626$ & $2501 \cdot 1332$ & 2265.0546 & $2084 \cdot 1218$ \\
\hline
\end{tabular}

A similar resolution was passed recommending that the I.A.U. adopt as secondary standards of wave-length the calculated wave-lengths of 30 neon lines as given in column 2 of Table I, on P. 205 of this volume, to replace the neon standards previously adopted.

The Commission passed a resolution recommending, also, that the I.A.U. adopt as secondary standards of wave-length the calculated wave-lengths of argon as given in 
column 2 of Tables 5 and 6,pp. 208 and 209 of this volume, with the exception of the wave-lengths in Table 5 marked ' $a$ '.

Swings reported that Migeotte had completed the preparation of 'An Atlas of the Infrared Solar Spectrum' covering the interval $2 \cdot 8 \mu$ to $23.75 \mu$. The wave-lengths have been measured, and most of the identifications completed. A copy of this Atlas was exhibited during the meeting.

Swings stated, also, that Prof. J. Genard of the Physical Institute of the University of Saarbrücken planned to carry out a programme on the measurement of all band spectra of astrophysical interest. To date, four sequences of the violet $\mathrm{CN}$ system, between $3500 \AA$ and $4700 \AA$, have been measured by Dr J. Weinard with an accuracy better than $\pm 0.0 \mathrm{I} A$, and the results will be published in $A n n$. d'Astroph. (I956). Part of the CN spectrograms were furnished by C. C. Kiess at the National Bureau of Standards; others were obtained by J. Genard and J. Weinard at the University of Saarbrücken. The study of the bands is in progress, and revised solar identifications have been made (to be published in $A n n$. d'Astroph.). Work will be continued on the red system of $\mathrm{CN}$ and on other cosmically abundant molecules.

As stated in the agenda of the General Assembly, resolution I4, item (a), p. 26, the Commission recommended the adoption of the symbol $\AA$ for the wave-length unit, to conform to the following motion which was adopted by the Joint Commission for Spectroscopy at the Lund meeting in I954:

Since the I.U.P.A.P., the I.A.P.A.C. and the Bureau International des Poids et Mesures have adopted $\AA$ as the symbol for the angstrom-unit and because this symbol has the great advantage over $A$ of being absolutely unique and characteristic, the J.C.S. recommends the universal use of the symbol $\AA$ for the angstrom-unit in all spectroscopic publications.

The Draft Report as a whole was approved.

In a letter to the President, O. C. Mohler had stated that the question of exçitation potentials had come up in connexion with the Michigan table of infra-red solar wavelengths. He writes 'It seems to us that it is no longer wise to publish excitation potentials in wave-length tables if these potentials have been derived from the spectroscopic term values. A much better procedure would be to publish the term values themselves.' Mrs Sitterly commented that opinions on this question are far from unanimous. She had sent a questionnaire to about ninety workers in various fields and received forty-eight replies. The majority favoured the retention of excitation potentials in volts, calculated with the conversion factor from $\mathrm{cm}^{-1}$ to volts, $0 \cdot 00012395$, adopted by the J.C.S. in I952.

The Commission felt that it should assume responsibility with regard to wave-length standards in the region I to $3 \mu$, and in the vacuum ultra-violet region. Edlen noted that the standards in the vacuum ultra-violet must be established by calculations based upon the combination principle. The Commission favoured extending the use of interferometric methods, including the use of the reflexion echelon, for measurements in both regions.

Meggers reported on the advantages of electrodeless high-frequency discharge lamps as sources for future spectroscopic wave-length standards, as follows:

For nearly half a century the iron arc at atmospheric pressure has been the major source of secondary standards. But this source emits lines greatly widened by Doppler effect (because the temperature is near $5000^{\circ} \mathrm{K}$.), and further broadened by collision and resonance-effects, or displaced (because of atmospheric pressure). Since these lines are relatively wide, their wave-lengths cannot be individually determined with high accuracy. The iron standards now computed with higher accuracy from mean values of atomic energy levels derived from interferometer measurements mark the culmination of work of this kind, but they do not increase the accuracy of measurement of individual lines. The only way to improve the secondary iron standards is to sharpen the lines by reducing the temperature and pressure of the source. Suggestions that this be done by operating the arc at reduced pressure or by substituting 
the hollow-cathode discharge have not been generally accepted. Recently, the development of electrodeless metal vapour lamps excited by microwaves (generated by magnetrons) has made available ideally simple sources of superior secondary standards. Since these sources operate at moderate temperatures, and very low pressures, the radiations are emitted with high intensity, but with greatly reduced Doppler width and relative freedom from pressure effects or self-reversal. Such quartz lamps containing a trace of iron halide, and $1-2 \mathrm{~mm}$. of argon gas, emit iron lines that produce interference patterns with four to five times the interference order obtainable with the standard arc.

The iron spectrum as a source of secondary standards has been criticized by spectroscopists working with very high dispersion because it does not permit the determination of standards throughout the spectrum at intervals of a few angstroms. Simple electrodeless ultra-highfrequency lamps containing thorium or uranium halides are suggested as sources of future secondary standards that will satisfy all foreseeable needs for making accurate descriptions of extremely complex spectra like those characterizing the rare-earth elements. Both 9oTh and $92 U$ occur naturally as practically pure even-mass isotopes ( 232 and 238 , respectively) and, therefore, emit lines of minimum Doppler width completely free of isotopic structure (and pressure shifts).

Jackson reported that he had developed and used since I928 a similar method of producing spectra with narrow lines from metals or metallic halides, by means of a high-frequency discharge in a carrier gas. The chief differences from Meggers' method were that $(I)$ the wave-length of the oscillator used for producing the discharge was much longer, approximately $30 \mathrm{~m}$.; and (2) the design of the discharge tubes was such that the vapour pressure of the metal or metallic halide could be controlled to any desired value, and was not determined by the heating effect of the discharge. The discharge tube must have fairly large end-pieces, with external electrodes in the form of thin copper foil wrapped around them. With the carrier gases helium or neon he has succeeded in observing the resonance lines of $\mathrm{Cs}, \mathrm{Rb}$, and $\mathrm{K}$ free from self-reversal. Similarly, by using thallous chloride, indium trichloride, and gallium tribromide, with the vapour pressure of the halides so high that the helium spectrum is almost extinguished, the resonance lines of the metals are quite free from the effects of self-reversal or self-absorption. With silver chloride precise control of temperature and vapour is necessary, as with the alkali metals, to avoid self-reversal. Jackson described, also, a very simple design for a cooled hollow cathode tube that could be constructed at little expense and quickly assembled.

The Commission recommended the investigation of the hollow cathode tube and the electrodeless discharge as sources for future work on secondary standards.

Engelhard discussed the merits of a primary standard of krypton. He described the $\mathrm{Kr}^{84}$ lamp developed by himself as an ideal source for the primary standard of wavelength.

Barrell felt that at present $\mathrm{Kr}^{84}$ was the best source, although $\mathrm{Hg}^{198}$ in a hollow cathode or an atomic beam may ultimately prove to be superior. He strongly recommended the adoption of a fixed path difference of $125 \mathrm{~mm}$., i.e. an etalon length of $62.5 \mathrm{~mm}$., in interferometric comparisons with the present primary standard. The adoption of one specific path difference simplifies procedure and eliminates from the results any small variations due to the effects of possible hyperfine structure in the cadmium red line.

Barrell submitted the following provisional interferometric vacuum wave-length measurements of $\mathrm{Hg}^{198}$ lines excited in water-cooled lamps at $20^{\circ} \mathrm{C}$.:

$5792 \cdot 2680$

5771.1979
$5462 \cdot 2702$
4359:5620

4348.7175
4078.9889

4047·7141 
Report of the meeting of Sub-commission I4a. 3I August I955

President: Prof. M. Minnaert.

SeCRetary: Prof. C. W. Allen.

The Draft Report of the sub-commission was approved.

The commission discussed a proposal by D. H. Menzel and D. Layzer that the single quantity line-strength $S$ be used for expressing intensities of spectrum lines. In the discussion that ensued, the advantages and disadvantages of using the quantity $S$ were tabulated as follows:

Advantages. $S$ is symmetric with respect to the initial and final level (although this applies also to $g f$ ). $S$ can be readily extrapolated along iso-electronic sequences. Multiplet sum-rules are more fundamental than $f$ sum-rules. Formulae relating to line and multiplet strengths may be simply derived from the strength of the whole multiplet.

Disadvantages. The $S$ unit is different for dipole, quadrupole and magnetic dipole radiations. There are some general quantum mechanical calculations, for example those depending on electron acceleration rather than electric moment, that are not related directly to $S$.

The alternatives suggested for $S$ were $g f, g f \lambda$, and $g f \lambda / \lambda_{0}$, where $g$ is statistical weight, $f$ oscillator strength, $\lambda$ wave-length and $\lambda_{\circ}$ a standard wave-length. It was decided that $f$ should not be used alone for this purpose (as it is unsymmetrical), but that the relative advantages of using $S, g f, g f \lambda$, and $g f \lambda / \lambda_{\circ}$ should be determined by usage during the next three years.

There was a discussion on the question of producing a general table of transition probabilities by the sub-commission. It was agreed that such a table would be of great use, but difficult problems of the contents and form suitable for such tables are involved.

C. W. Allen gave a brief account of the differences between calculated and observed oscillator strengths for lines of the Fe group of atoms. The discrepancies are very closely related to the excitation potential. 